\title{
ON CERTAIN SUBSETS OF FINITE BOOLEAN ALGEBRAS
}

\section{K. DURST}

1. The boolean algebra $B_{n}$, of finite dimension $n$, may be written as the direct union $B_{1} \times B_{1} \times \cdots \times B_{1}$ of $n$ copies of $B_{1}$. Consequently each element $u$ of $B_{n}$ may be represented by an $n$-digit binary number. Let $G_{n}$ be the group of those permutations on the elements of $B_{n}$ which interchange or invert various of the factors in the direct union expansion. Thus the elements of $G_{n}$ permute the components of the $u$ or interchange 0,1 in certain components of every $u$ in $B_{n}$. The order of $G_{n}$ is therefore $2^{n} n$ !.

Two subsets of $B_{n}$ will be called congruent modulo $G_{n}$ if one is the image of the other under transformation by an element of $G_{n}$. Clearly sets congruent modulo $G_{n}$ have the same number of elements. The number of elements in a subset will be called the order of the subset. Let $N_{n}^{(s)}$ be the number of congruence classes of subsets of order $s$. Note that $N_{n}^{(s)}=N_{n}^{\left(2^{n}-s\right)}$. Pólya [1] has calculated $N_{n}^{(s)}$ for $0 \leqq s \leqq 2^{n}$ and $n=1,2,3,4$, and Slepian [2] has found the values of $N_{n}=\sum_{s=0}^{2^{n}} N_{n}^{(s)}$ for $n=5$, 6. Trivially, $N_{n}^{(0)}=N_{n}^{(1)}=1$, all $n$; and it is almost as obvious that $N_{n}^{(2)}=n$, all $n \geqq 1$ (see $\S 2$ ).

In this note an elementary argument is given which yields an explicit expression for $N_{n}^{(3)}$ good for all $n>1$ (Theorem 2).

2. The procedure for calculating $N_{n}^{(3)}$ is based on the notion of the "dimension" of a subset of $B_{n}$. Let $S$ be a subset of $B_{n}$ whose order is at least 2. Let $\vee S$ and $\wedge S$ be the lattice-union and lattice-intersection, respectively, of the elements of $S$. (The symbols $\vee, \wedge$, and $\leqq$ will be used for the lattice operations and the ordering relation in $B_{n}$, while $\cup, \cap$, and $\subseteq$ will be reserved for their set-theoretical counterparts.) Since the quotient $\vee S / \wedge S$ is relatively complemented, it is a boolean algebra, say $B_{r}$ if of dimension $r . B_{r}$ may be called the connected closure of $S$. The relation between a subset $S$ and its connected closure will be written: $S<B_{r}$. The dimension of $S$ is defined to be the dimension of $B_{r}$ if $S \prec B_{r}$. Thus $S<B_{r}$ implies $\operatorname{dim} S=r$. If the order of $S$ is two, $\operatorname{dim} S$ is simply the usual metric in $B_{n}$.

The technique to be used for counting incongruent sets in $B_{n}$ is to determine the number of incongruent sets of given order having maximal dimension in $B_{k}$ for each $k \leqq n$. Thus, for instance, $N_{n}^{(2)}=n$,

Presented to the Society, December 29, 1954, under the title On some numbers of $P$ olya; received by the editors November 4,1954 . 
all $n \geqq 1$; for in $B_{k}$ all sets of order two and maximal dimension are congruent.

Lemma. Suppose $S$ is a subset of $B_{n}$ of order 3 or more. If $S=\{u\} \cup T$ and $T<B_{r}$, then $S$ has maximal dimension if and only if $u \in B_{r}^{\prime}$ where $B_{r}^{\prime}$ is the set of the complements of the elements of $B_{r}$.

Proof. If $u \in B_{r}^{\prime}$, then $u \wedge(\wedge T) \leqq u \wedge u^{\prime}=0$ and $u \vee(\vee T)$ $\geqq u \vee u^{\prime}=I$, i.e. $S=\{u\} \cup T$ has maximal dimension. But if $u \notin B_{r}^{\prime}$, and $v \in B_{r}$ then either $u \wedge v>0$ or $u \vee v<I$, since complements are unique, and in this case $\operatorname{dim} S$ cannot be maximal.

3. Consider now the case $s=3$ and suppose $S=\left\{u_{1}, u_{2}, u_{3}\right\}$. Let $r_{i}=\operatorname{dim}\left\{u_{j}, u_{k}\right\}$, where $i, j, k$ is some permutation of $1,2,3$. Clearly $1 \leqq r_{i} \leqq n$.

THEOREM 1. $\operatorname{dim}\left\{u_{1}, u_{2}, u_{3}\right\}=n$ if and only if $r_{1}+r_{2}+r_{3}=2 n$.

Proof. If $u_{i}=x_{1}^{i} x_{2}^{i} \cdots x_{n}^{i}, 1 \leqq i \leqq 3$, are the binary representations of the $u_{i}$, no generality is lost by assuming $x_{i}^{1}=1,1 \leqq i \leqq n ; x_{i}^{2}=1$, $1 \leqq i \leqq n-r_{3} ; x_{i}^{2}=0, n-r_{3}+1 \leqq i \leqq n$. Suppose further that $x_{i}^{3}=0$, $1 \leqq i \leqq k$ and $n-r_{3}+1 \leqq i \leqq n-l ; x_{1}^{3}=1, k+1 \leqq i \leqq n-r_{3}$ and $n-l+1$ $\leqq i \leqq n$; where $k \leqq n-r_{3}, l \leqq r_{3}$. The lemma implies that $\operatorname{dim}\left\{u_{1}, u_{2}, u_{3}\right\}$ $=n$ if and only if $k=n-r_{3}$. But $r_{1}=k+l, r_{2}=k+r_{3}-l$, so that $r_{1}+r_{2}+r_{3}=2\left(k+r_{3}\right)$; from which the theorem follows.

The expressions for the binary components also give the following immediate corollary.

Corollary. If $\left\{u_{1}, u_{2}, u_{3}\right\},\left\{v_{1}, v_{2}, v_{3}\right\}$ are subsets of $B_{n}$ with maximal dimension, and if

$$
\operatorname{dim}\left\{u_{i}, u_{j}\right\}=\operatorname{dim}\left\{v_{i}, v_{j}\right\}, \quad 1 \leqq i, j \leqq 3,
$$

then $\left\{u_{1}, u_{2}, u_{3}\right\}$ and $\left\{v_{1}, v_{2}, v_{3}\right\}$ are congruent modulo $G_{n}$.

Theorem 1 and the corollary imply that the number of incongruent sets of order 3 having maximal dimension in $B_{n}$ is precisely the number of solutions of the following diophantine system:

$$
2 n=x+y+z, \quad 1 \leqq x \leqq y \leqq z \leqq n .
$$

THEOREM 2.

$$
N_{n}^{(3)}=\sum_{k=2}^{n}\left\{\left[\frac{k}{3}\right]+\sum_{r=0}^{[k / 3]}\left[\frac{k-3 r}{2}\right]\right\} .
$$

Proof. For each $k, 2 \leqq k \leqq n$, it suffices to count the number of solutions, $N_{k}^{(3)}-N_{k-1}^{(3)}$, of 


$$
2 k=x+y+z, \quad 1 \leqq x \leqq y \leqq z \leqq k .
$$

Suppose $z=k-r$, then $0 \leqq r \leqq[k / 3]$ since $z=2 k-x-y \geqq 2 k-2 z$, or $3 z \geqq 2 k$. Now $y \leqq z=k-r$ and $x=k+r-y \geqq k+r-(k-r)=2 r$, but $x \leqq(k+r) / 2$ so $2 r \leqq x \leqq(k+r) / 2$. For $r=0$, there are $[k / 2]$ possible values for $x$; and if $r>0$, there are $[(k+r) / 2-(2 r-1)]$. Hence

$$
\begin{aligned}
N_{k}^{(3)}-N_{k-1}^{(3)}= & {\left[\frac{k}{2}\right]+\sum_{r=1}^{[k / 3]}\left[\frac{k-3 r+2}{2}\right] } \\
= & -1+\sum_{r=0}^{[k / 3]}\left[\frac{k-3 r+2}{2}\right] \\
= & {\left[\frac{k}{3}\right]+\sum_{r=0}^{[k / 3]}\left[\frac{k-3 r}{2}\right] . } \\
& \text { REFERENCES }
\end{aligned}
$$

1. G. P6lya, J. Symbolic Logic, vol. 5 (1940) pp. 98-103.

2. D. Slepian, Canadian Journal of Mathematics vol. 5 (1953) pp. 185-193.

Bell TElephone Laboratories aNd

THE RICE INSTITUTE 